\title{
Relativistic Multipoles and the Advance of the Perihelia
}

\author{
Bruno Boisseau' and Patricio S. Letelier巳̈, \\ Laboratoire de Mathématiques et Physique Théorique \\ CNRS/UMR 6083, Université François Rabelais \\ Faculté des Sciences et Techniques \\ Parc de Grandmont 37200 TOURS, France
}

\begin{abstract}
In order to shed some light in the meaning of the relativistic multipolar expansions we consider different static solutions of the axially symmetric vacuum Einstein equations that in the non relativistic limit have same Newtonian moments. The motion of test particles orbiting around different deformed attraction centers with the same Newtonian limit is studied paying special attention to the advance of the perihelion. We find discrepancies in the fourth order of the dimensionless parameter (mass of the attraction center)/(semilatus rectum). An evolution equation for the difference of the radial coordinate due to the use of different general relativistic multipole expansions is presented.
\end{abstract}

PACS numbers: $04.20 \mathrm{Jb}, 04.25 \mathrm{Nx}, 95.10$ ce, 04.70 BW

\footnotetext{
${ }^{1}$ e-mail: boisseau@celfi.phys.univ-tours.fr

${ }^{2}$ e-mail: letelier@ime.unicamp.br

${ }^{3}$ Permanent address: Departamento de Matemática Aplicada-IMECC, Universidade Estadual de Campinas, 13083-970 Campinas. S.P., Brazil
} 


\section{Introduction.}

The adequate description of the gravitation field of an astrophysical object has been an important subject in both relativistic and Newtonian gravity since their origins. The particular case of the gravity associated to axially symmetric bodies has played a central role in this discussion. Recently, Merrit [1] found, from detailed modeling of triaxial galaxies, that most of the galaxies must be nearly axisymmetric, either prolate or oblate. In Newtonian theory the gravitational potential of axially symmetric bodies can be always represented by its usual expansion in terms of Legendre polynomials (zonal harmonics). In general relativity we have that the solution of the vacuum Einstein equations associated to static axially symmetric bodies has a simple form with only two metric functions [2].

As a consequence of the self interaction of the general relativistic gravitational field we have a rather elaborate description of multipolar fields, the covariant multipole moments [3]. For the special case of axially symmetric spacetimes we have geometric properties that can also be used to define a class of multipole moments [4, 5]. It is highly non trivial to prove the equivalence between these two definitions [6]. Also, due to the nonlinearity of the Einstein equations we do not have a simple expansion of the metric in terms of relativistic multipoles like the expansion of the potential in Newtonian gravity. Moreover, it is not know how to construct a solution to the vacuum Einstein equation with assigned relativistic multipoles. Also the physical consequences of having different relativistic moments are rather obscure. Furthermore, the problem to model a static axially symmetric center of attraction in general relativity with some multipolar perturbations that have a prescribed Newtonian limit is not a well define problem, as a matter of 
fact we have an infinite number of solutions of the vacuum Einstein equations with the same Newtonian limit. This problem arises in a natural form when one studies the orbits in deformed centers of attraction, see for instance [7].

In this paper, in order to shed some light in the physical meaning of the relativistic multipolar expansions, we consider different static solutions of the axially symmetric vacuum Einstein equations that in the non relativistic limit have same Newtonian moments. The particular solutions considered represent a large class of rather natural solutions that are generalizations of some well know ones.

Along this article we shall use the terminology "distorted black hole" introduced in [8] to indicate an attraction center with multipolar moments, examples are: a) A true black hole (or a dense object) surrounded by a distribution of matter like a ring or a small disk formed by counterrotating matter, i.e., built by approximately the same number of particles moving clockwise as counterclockwise. Even though, this interpretation can be seen as a device to have a static stable configuration there are observational evidence of disks made of streams of rotating and counterrotating matter [9]. b) An axially symmetric static dense object with either polar deformations or polar jets. In our analysis the horizon will play no role, therefore our results will be valid for any nearly static axially symmetric attraction center.

Since the advance of the perihelion is one of the most significant features of the general relativistic celestial mechanics will be considered in some detail. We find discrepancies in the fourth order of the dimensionless parameter (mass of the attraction center)/(semilatus rectum). We present an evolution equation for the difference of the radial coordinate due to the use of different general relativistic multipole expansions.

In Sec. 2 we present a summary of the main expressions associated to 
the Weyl solutions. In Sec. 3 we study the Newtonian limit of four different solutions to the Einstein equations. Also we study the relativistic multipoles moments for each solution. These solutions are the Erez-Rosen-Quevedo (ERQ) solution [10], a solution characterized by the usual Newtonian multipoles and generalizations of both. We present two graphics that show the difference between the solutions. In the next section, Sec. 4, we study series expansions of the solutions restricted to the equatorial plane. Also several differences of the metric potentials are considered. In Sec. 5 an evolution equation for the difference of the radial coordinate due to the use of different multipole expansions is presented. We solve this equation for circular orbits in the equatorial plane. In Sec. 6, the perihelion shift is computed to the order in which the first discrepancies appears due to the use of the different multipolar expansions. As a particular case we have the perihelion shift in a Schwarzschild spacetime up to a fourth order. We also solve the difference equation of the preceding section for generic orbits on the equatorial plane. Finally, in Sec. 7, we present a short discussion of our main results.

\section{Static axially symmetric solutions of Ein- stein vacuum equations.}

The external gravitational field of produced by an axially symmetric body can be well described by the Weyl metric [2],

$$
d s^{2}=e^{2 \psi} d t^{2}-e^{-2 \psi}\left[r^{2} d \varphi^{2}+e^{2 \gamma}\left(d r^{2}+d z^{2}\right)\right]
$$

where the functions $\psi$ and $\gamma$ depend only on $r$ and $z$; the ranges of the coordinates $(r, \varphi, z)$ are the usual for cylindrical coordinates and $-\infty<t<$ $+\infty$. The vacuum Einstein equations $\left(R_{\mu \nu}=0\right)$ reduces to the usual Laplace 
equation in cylindrical coordinates,

$$
\psi_{, r r}+\psi_{, r} / r+\psi_{, z z}=0
$$

and the quadrature,

$$
d \gamma[\psi]=r\left[\left(\psi_{, r}^{2}-\psi_{, z}^{2}\right) d r+2 \psi_{, r} \psi_{, z} d z\right]
$$

When $\psi$ satisfies the Laplace equation this differential is exact.

The Schwarzschild solution in Weyl coordinates takes the form

$$
\begin{aligned}
\psi_{S} & =\frac{1}{2} \ln \frac{R_{+}+R_{-}-2 m}{R_{+}+R_{-}+2 m}, \\
\gamma_{S} & =\frac{1}{2} \ln \frac{\left(R_{+}+R_{-}\right)^{2}-4 m^{2}}{R_{+} R_{-}},
\end{aligned}
$$

where

$$
R_{ \pm}=\sqrt{r^{2}+(z \pm m)^{2}} .
$$

The function $\psi_{S}$ is just the Newtonian potential of a bar of length $2 m$ and density $1 / 2$. The fact that in Weyl coordinates the Schwarzschild metric does not look spherically symmetric and that the horizon is squeezed into a line of length $2 m$ has prevented the wide use of these coordinates. Since the superposition of static axially symmetric perturbation can be performed in a simple way and exact results can be obtained, we believe that is worth to use these coordinates. More suitable coordinates are the spheroidal coordinates,

$$
x=\left(R_{+}+R_{-}\right) /(2 m), y=\left(R_{+}-R_{-}\right) /(2 m) .
$$

Note that the coordinates $(x, y)$ are dimensionless and $1 \leq x<\infty$ and $-1 \leq y \leq 1$. The coordinate $x$ is essentially a radial coordinate and $y$ the cosine of an angle. The inverse of (17) are,

$$
r=m \sqrt{\left(x^{2}-1\right)\left(1-y^{2}\right)}, \quad z=m x y .
$$


In this case the Weyl metric takes the form,

$d s^{2}=e^{2 \psi} d t^{2}-m^{2} e^{-2 \psi}\left[e^{2 \gamma}\left(x^{2}-y^{2}\right)\left(\frac{d x^{2}}{x^{2}-1}+\frac{d y^{2}}{1-y^{2}}\right)+\left(x^{2}-1\right)\left(1-y^{2}\right) d \varphi^{2}\right]$

and the Einstein equations,

$$
\begin{aligned}
& {\left[\left(1-x^{2}\right) \psi_{, x}\right]_{, x}+\left[\left(y^{2}-1\right) \psi_{, y}\right]_{, y}=0} \\
& \gamma_{, x}=\frac{1-y^{2}}{x^{2}-y^{2}}\left[x\left(x^{2}-1\right) \psi_{, x}^{2}-x\left(1-y^{2}\right) \psi_{, y}^{2}-2 y\left(x^{2}-1\right) \psi_{, x} \psi_{, y}\right] \\
& \gamma_{, y}=\frac{x^{2}-1}{x^{2}-y^{2}}\left[y\left(x^{2}-1\right) \psi_{, x}^{2}-y\left(1-y^{2}\right) \psi_{, y}^{2}+2 y\left(1-y^{2}\right) \psi_{, x} \psi_{, y}\right] .
\end{aligned}
$$

In this coordinates the Schwarzschild solutions takes the particularly simple form

$$
\begin{aligned}
\psi_{S} & =-Q_{0}(x) \\
& =\frac{1}{2} \ln \frac{x-1}{x+1} \\
\gamma_{S} & =\frac{1}{2} \ln \frac{x^{2}-1}{x^{2}-y^{2}} .
\end{aligned}
$$

From the coordinate transformations, $x=R_{s} / m-1, \quad y=\cos \vartheta$, and (9) with (13) and (14) we recover the Schwarzschild metric in its usual form,

$$
d s^{2}=\left(1-2 m / R_{s}\right) d t^{2}-\left(1-2 m / R_{s}\right)^{-1} d R_{s}^{2}-R_{s}^{2}\left(d \vartheta^{2}+\sin \vartheta^{2} d \varphi^{2}\right) .
$$

We shall consider solutions of the form,

$$
\psi=\psi_{S}+\hat{\psi}
$$

where $\hat{\psi}(x, y)$ represents the superposition of external multipolar fields solutions of Laplace equation with no monopolar term. The function $\gamma$ in this case can be written as

$$
\gamma[\psi]=\gamma_{S}+\hat{\gamma}
$$


with

$$
\begin{aligned}
& \hat{\gamma}=2 \gamma\left[\psi_{S}, \hat{\psi}\right]+\gamma[\hat{\psi}] \\
& d \gamma\left[\psi_{S}, \hat{\psi}\right]=r\left[\left(\psi_{S, r} \hat{\gamma}_{, r}-\psi_{S, z} \hat{\gamma}_{, z}\right) d r+\left(\psi_{S, r} \hat{\gamma}_{, z}+\psi_{S, z} \hat{\gamma}_{, r}\right) d z\right]
\end{aligned}
$$

In this case the Weyl metric takes the form,

$$
d s^{2}=\left(1-\frac{2 m}{R_{S}}\right) e^{2 \hat{\psi}} d t^{2}-\frac{e^{2(\hat{\gamma}-\hat{\psi})}}{1-\frac{2 m}{R_{S}}} d R_{S}^{2}-R_{S}^{2} e^{2(\hat{\gamma}-\hat{\psi})} d \vartheta^{2}-R_{S}^{2} e^{-2 \hat{\psi}} \sin ^{2} \vartheta d \varphi^{2}
$$

that has been interpreted as a "black hole" with multipolar deformations [8]

Note that this metric for a given function $\hat{\psi}$ - solution of the usual axially symmetric Laplace equation - and its associated potential $\hat{\gamma}$ given by Eq. (18) is an exact solution of the Einstein vacuum field equations. We shall consider several different classes of approximations. In principle, since we start with an exact framework we will be able to control the validity of the approximations. The relation of this Weyl approach in the case linear perturbations with the Regge-Wheeler [11] formalism - general linear perturbations of a black hole - can be found in [12]. In the present paper, in some cases, we will go beyond the linear case.

\section{Multipoles and multipolar fields.}

In order to gain some understanding of the solutions of the vacuum Einstein equations with different relativistic moments, in this section, we study different Weyl solutions that represent static attraction centers that have the same Newtonian limit.

Following Ehlers [14], for a given Weyl solution we define its Newtonian limit as $\lim _{\lambda=0} \psi(\lambda, r, z) / \lambda$, where $\lambda \equiv c^{-2}$ and $c$ the light velocity, e.g. 
for the Schwarzschild solution we put $m=\lambda G M$ in (四) and with the aid of l'Hôpital rule we get

$$
\lim _{\lambda=0} \psi_{S}(\lambda, r, z) / \lambda=-G M / R
$$

with

$$
R=\sqrt{r^{2}+z^{2}}
$$

There are an infinite number of different Weyl solutions that represent fields of multipoles with the same Newtonian multipolar limit. We shall restrict our study to two known solutions that have been used recently in some applications and other two that are generalizations of the formers. They are:

a) The usual Newtonian multipolar fields,

$$
\hat{\psi}^{N}=\sum_{k=1}^{\infty} q_{k} \frac{P_{k}(z / R)}{R^{k+1}}
$$

we have the same $\hat{\psi}^{N}$ as its Newtonian limit. Note that $q_{k}$ scales with $\lambda$ as $\lambda q_{k}$.

b) Separating variables in (10) one finds the ERQ solution 15, 10,

$$
\hat{\psi}^{E R Q}=\sum_{k=1}^{\infty} \frac{(2 k+1) !}{2^{k}(k !)^{2} m^{k+1}} q_{k} Q_{k}(x) P_{k}(y)
$$

where $Q_{k}(x)$ and $P_{k}(y)$ are the Legendre functions of the second kind and the Legendre polynomials, respectively. We have chosen the coefficients in the series to have

$$
\lim _{\lambda=0} \psi_{n}^{E R Q}(\lambda, r, z) / \lambda=\psi_{n}^{N},
$$

where $\psi_{n}^{E R Q}$ and $\psi_{n}^{N}$ are the $n$-term in the series (24) and (23), respectively. To find the limit is useful the Letelier identity [16],

$$
Q_{n}(x) P_{n}(y)=\frac{1}{2} \int_{-m}^{m} \frac{P_{n}(\alpha / m)}{\sqrt{r^{2}+(z-\alpha)^{2}}} d \alpha
$$


c) The solutions of Laplace equation:

$$
\begin{aligned}
& \hat{\psi}^{I}=\sum_{k=1}^{\infty} q_{k}\left[\alpha \frac{P_{n}\left((z+a m) / R_{+a}\right)}{R_{+a}^{k+1}}+(1-\alpha) \frac{P_{n}\left((z-a m) / R_{-a}\right)}{R_{-a}^{k+1}}\right] \\
& \hat{\psi}^{I I}=\sum_{k=1}^{\infty} \frac{(2 k+1) !}{2^{k}(k !)^{2}(m a)^{k+1}} q_{k} Q_{k}\left(x_{a}\right) P_{k}\left(y_{a}\right)
\end{aligned}
$$

where

$$
\begin{aligned}
& x_{a}=\left(R_{+a}+R_{-a}\right) /(2 m a), \quad y_{a}=\left(R_{+a}-R_{-a}\right) /(2 m a), \\
& R_{+a}=\sqrt{r^{2}+(z+a m)^{2}}, \quad R_{-a}=\sqrt{r^{2}+(z-a m)^{2}}
\end{aligned}
$$

$\alpha$ and $a$ are arbitrary constants that we shall take as positive and less than one, we shall comeback to this point later. $\hat{\psi}^{I}$ is obviously a solution of the Laplace equation. To prove that $\hat{\psi}^{I I}$ is also a solution of this last equations one can use the identity (26). $\hat{\psi}^{I}$ and $\hat{\psi}^{I I}$ are nontrivial deformations of the Newtonian multipoles and the ERQ solution, respectively. Note that in the superposition (16) the Schwarzschild term $\psi_{S}$ is not changed. Letting $a=0$ in $\psi^{I}$ we get $\psi^{N}$, also putting $a=1$ in $\psi^{I I}$ we recover $\psi^{E R Q}$. The global properties of these new solutions will be presented elsewhere.

The first two solutions are the axially symmetric multipolar expansion for the Newtonian Laplace equation in spherical coordinates (usual expansion) and in spheroidal prolate coordinates, respectively. The second one has the property of having the monopolar term proportional to the Weyl potential that gives rise to the Schwarzschild solution [cf. Eq. (13)]. The Schwarzschild solution with multipolar deformations represented by either expansions have been study by several authors (see for instance, [10, 17]), specially in the important case of quadrupolar deformations [8, 15]. For recent applications see [0, 18]. The last two multipolar expansions, $\hat{\psi}^{I}$ and $\hat{\psi}^{I I}$, are new ones and are closed related to the previous ones. They have some 
new parameters that do not appear in the Newtonian limit. They will be considered in order to explore the possibility to have relativistic multipoles not completely determined by its Newtonian ones. We shall comeback to this point later.

The relativistic multipoles $m_{k}$ for a Weyl solution can be computed from the function $\psi$ evaluated on the axis of symmetry, in the following way [10, 13]: First we define the function

$$
\xi(x, y)=\frac{1-e^{2 \psi(x, y)}}{1+e^{2 \psi(x, y)}}
$$

and find its value on the axis of symmetry $r=0$, i.e., $y=1$; so $x=z / m$. Then we calculate the quantities

$$
\bar{m}_{k}=\left.\frac{1}{(k+1) !} \frac{d^{k+1} \xi(\tilde{z}, 1)}{d \tilde{z}^{k+1}}\right|_{\tilde{z}=0}
$$

with $\tilde{z}=1 / z$. The relativistic moments are given by

$$
m_{k}=\bar{m}_{k}+d_{k}
$$

where the first six constants $d_{k}$ are 13 :

$$
\begin{aligned}
& d_{0}=d_{1}=d_{2}=d_{3}=0, \\
& d_{4}=\bar{m}_{0}\left(\bar{m}_{1}^{2}-\bar{m}_{2} \bar{m}_{0}\right) / 4, \\
& d_{5}=\bar{m}_{0}\left(\bar{m}_{2} \bar{m}_{1}-\bar{m}_{3} \bar{m}_{0}\right) / 3+\left(\bar{m}_{1}\left(\bar{m}_{1}^{2}-\bar{m}_{2} \bar{m}_{0}\right) / 21 .\right.
\end{aligned}
$$

The direct computations of the relativistic multipolar moments up to the sixteenth pole gives:

a) For the Newtonian multipolar field $\hat{\psi}^{N}$,

$$
\begin{aligned}
& m_{0}=m, \quad m_{1}=q_{1}, \quad m_{2}=q_{2}, \quad m_{3}=q_{3}-m^{2} q_{1}, \\
& m_{4}=q_{4}-\left(8 m^{2} q_{2}+6 m q_{1}^{2}\right) / 7 .
\end{aligned}
$$


b) For the ERQ multipolar field $\hat{\psi}^{E R Q}$,

$$
\begin{aligned}
& m_{0}=m, \quad m_{1}=q_{1}, \quad m_{2}=q_{2}, \quad m_{3}=q_{3}-2 m^{2} q_{1} / 5, \\
& m_{4}=q_{4}-2\left(m^{2} q_{2}+3 m q_{1}^{2}\right) / 7 .
\end{aligned}
$$

c) For the multipolar field $\hat{\psi}^{I}$,

$$
\begin{aligned}
m_{0}= & m, \quad m_{1}=q_{1}, \quad m_{2}=q_{2}+2(1-2 \alpha) a m q_{1}, \\
m_{3}= & q_{3}+\left(3 a^{2}-1\right) m^{2} q_{1}+3 a(1-2 \alpha) m q_{2} \\
m_{4}= & q_{4}+4 a\left(7 a^{2}-4\right)(1-2 \alpha) m^{3} q_{1} / 7 \\
& +4(1-2 \alpha) a m q_{3}+2\left(21 a^{2}-4\right) m^{2} q_{2} / 7-6 m q_{1}^{2} / 7 .
\end{aligned}
$$

d) For the multipolar field $\hat{\psi}^{I I}$

$$
\begin{aligned}
& m_{0}=m, \quad m_{1}=q_{1}, \quad m_{2}=q_{2}, \quad m_{3}=q_{3}-\left(1-3 a^{2} / 5\right) m^{2} q_{1}, \\
& m_{4}=q_{4}-6 m q_{1}^{2} / 7-2\left(4-3 a^{2}\right) m^{2} q_{2} / 7 .
\end{aligned}
$$

Since our main goal is to study the physical effect of having different relativistic multipole moments is instructive to compute the differences of these multipoles for the above mentioned, solutions. We shall compute the quantities,

$$
\Delta m_{k}=m_{k}-m_{k}^{N} .
$$

We find for the ERQ, I and II solutions $\Delta m_{0}=\Delta m_{1}=0$, and

a) For the ERQ solution:

$$
\Delta m_{2}=0, \quad \Delta m_{3}=3 m q_{1} / 5, \quad \Delta m_{4}=6 m^{2} q_{2} / 7 .
$$

b) For the solution I:

$$
\begin{aligned}
& \Delta m_{2}=2 a(1-2 \alpha) m q_{1}, \quad \Delta m_{3}=3 a^{2} m^{2} q_{1}+3 a(1-2 \alpha) m q_{2}, \\
& \Delta m_{4}=4 a(1-2 \alpha)\left(7 a^{2}-4\right) m^{3} q_{1} / 7+6 a^{2} m^{2} q_{2}+4 a(1-2 \alpha) m q_{3} .
\end{aligned}
$$


c) For the solution II:

$$
\Delta m_{2}=0, \quad \Delta m_{3}=3 a^{2} m^{2} q_{1} / 5, \quad \Delta m_{4}=6 a^{2} m^{2} q_{2} / 7 .
$$

Along all the paper we shall compare the different solutions with $\psi^{N}$, of course this choice is arbitrary.

Since the quadrupolar strength $q_{2}$ plays an important role in the above differences - moreover when $q_{1}=0$ - we shall examine closely the quadrupolar potentials. A significant quantity is the quadrupolar deviation,

$$
\left(\psi_{2}-\psi_{2}^{N}\right) / \psi_{2}^{N}
$$

that we shall study graphically.

In Fig. 1 we present the deviation of $\psi_{2}=\psi_{2}^{I}$ on the plane $\vartheta=\pi / 2$ for $a=1$, and different values of the constant $\alpha$ : 0.25 (top curve), 0.5, 0.75, 1.0 (bottom). We can have large deviations that asymptotically vanish. In Fig. 2 we show the deviation $\psi_{2}=\psi_{2}^{I I}$ on the plane $\vartheta=\pi / 2$ for different values of the parameter $a$ : 0.25 (top curve), 0.5, 0.75, 1.0 (bottom). The case $a=1$ corresponds to the ERQ solution. It is not difficult to show using Letelier identity that in the limit $a=0, \psi^{I I}=\psi^{N}$, in other words the ERQ solution can continuously deformed in to $\psi^{N}$, fact that is confirmed in Fig. 2 for the quadrupolar term.

\section{Solutions and differences.}

For our purposes, it is enough to consider the the multipolar series only up to the fourth order term, $\psi_{4}$. Since one of our objectives is the study of orbits of test particles moving around a deformed center of attraction, the inverse "radial coordinate", $u=R_{S}^{-1}=[m(1+x)]^{-1}$, will be useful. Moreover, all 
the metric functions will be computed in the plane $\vartheta=\pi / 2$ up to the sixth order in $\mathrm{u}$; this order will be the adequated for consistence in the expansions. Also we shall disregard the dipolar moment, $q_{1}=0$ (we shall comeback to this point latter). We find for the different functions $\hat{\psi}=\hat{\psi}_{2}+\hat{\psi}_{3}+\hat{\psi}_{4}$ :

$$
\begin{aligned}
& \hat{\psi}^{N}=-\frac{1}{2} q_{2} u^{3}-\frac{3}{2} m q_{2} u^{4}-\frac{3}{8}\left(10 m^{2} q_{2}-q_{4}\right) u^{5} \\
& -\frac{5}{8} m\left(14 m^{2} q_{2}-3 q_{4}\right) u^{6} \\
& \hat{\psi}^{E R Q}=-\frac{1}{2} q_{2} u^{3}-\frac{3}{2} m q_{2} u^{4}-\frac{3}{56}\left(64 m^{2} q_{2}-7 q_{4}\right) u^{5} \\
& -\frac{5}{56} m\left(80 m^{2} q_{2}-21 q_{4}\right) u^{6} \\
& \hat{\psi}^{I}=-\frac{1}{2} q_{2} u^{3}-\frac{3}{2} m q_{2} u^{4}-\frac{3}{8}\left[2\left(5-3 a^{2}\right) m^{2} q_{2}+4(2 \alpha-1) a q_{3}-q_{4}\right] u^{5} \\
& -\frac{5}{8} m\left[2\left(7-9 a^{2}\right) m^{2} q_{2}+60(2 \alpha-1) a m q_{3}-3 q_{4}\right] u^{6}, \\
& \hat{\psi}^{I I}=-\frac{1}{2} q_{2} u^{3}-\frac{3}{2} m q_{2} u^{4}-\frac{3}{56}\left[\left(70-6 a^{2}\right) m^{2} q_{2}-7 q_{4}\right] u^{5} \\
& -\frac{5}{56} m\left[2\left(49-9 a^{2}\right) m^{2} q_{2}-21 q_{4}\right) u^{6} .
\end{aligned}
$$

The respective functions $\hat{\gamma}$ are:

$$
\begin{aligned}
& \hat{\gamma}^{N}=-\frac{3}{4} u^{4}-3 m^{2} q_{2} u^{5}-\frac{1}{8}\left(3 q_{2}^{2}+70 m^{3} q_{2}-5 m q_{4}\right) u^{6} \\
& \hat{\gamma}^{E R Q}=-\frac{3}{4} u^{4}-3 m^{2} q_{2} u^{5}-\frac{1}{56}\left(21 q_{2}^{2}+460 m^{3} q_{2}-35 m q_{4}\right) u^{6}, \\
& \hat{\gamma}^{I}=-\frac{3}{4} u^{4}-3 m^{2} q_{2} u^{5}-\frac{1}{8}\left(3 q_{2}^{2}+70 m^{3} q_{2}-30 a^{2} m^{3} q_{2}-5 m q_{4}\right) u^{6}, \\
& \hat{\gamma}^{I I}=-\frac{3}{4} u^{4}-3 m^{2} q_{2} u^{5}-\frac{1}{56}\left(21 q_{2}^{2}+490 m^{3} q_{2}-30 a^{2} m^{3} q_{2}\right. \\
& \left.-35 m q_{4}\right) u^{6}
\end{aligned}
$$

The exact function $\gamma$ for the Schwarzschild solution with Newtonian multipoles can be found explicitly for the general case [17], and in the case of the multipoles $\hat{\psi}^{E R Q}$ in [10]. These relations are rather formidable and of not 
much use. An integral representation of the function $\gamma$ for the ERQ solution can be found in [16]. For the solution $I$ we were able to find $\gamma$ in the general case. For the solution $I I$ up to the quadrupolar moment. These results are rather cumbersome an will be presented elsewhere.

Note that to eliminate conic singularities along the axis of symmetry we need to impose the boundary condition $\lim _{r=0} \gamma=0$ outside the source. The solutions with this boundary condition are asymptotically flat.

Since our main goal is to study different solutions with the same Newtonian multipole moments we shall compute the quantities,

$$
\delta \psi=\psi-\psi^{N}, \quad \delta \gamma=\gamma-\gamma^{N}
$$

Note that $\delta \psi=\delta \hat{\psi}$ and $\delta \gamma=\delta \hat{\gamma}$. We find,

$$
\begin{gathered}
\delta \psi^{E R Q}=\frac{9}{28} m^{2} q_{2} u^{5}+\frac{45}{28} m^{3} q_{2} u^{6}, \\
\delta \psi^{I}=\frac{3}{4} a\left[3 a m q_{2}-2(2 \alpha-1) q_{3}\right] m u^{5} \\
\quad+\frac{15}{4} a\left[3 a m q_{2}-2(2 \alpha-1) q_{3}\right] m^{2} u^{6}, \\
\delta \psi^{I I}=a^{2} \delta \psi^{E R Q} .
\end{gathered}
$$

And for the function $\gamma$,

$$
\begin{aligned}
& \delta \gamma^{E R Q}=\frac{15}{28} m^{3} q_{2} u^{6} \\
& \delta \gamma^{I}=\frac{5}{4} a\left[3 a m q_{2}-2(2 \alpha-1) q_{3}\right] m^{2} u^{6} \\
& \delta \gamma^{E R Q}=\frac{15}{28} a^{2} m^{3} q_{2} u^{6}
\end{aligned}
$$

These quantities will play and essential role in our analysis, we note that the quadrupolar strength is the main parameter that appears in these differences. Also in $\delta \psi^{I}$ and $\delta \gamma^{I}$ we have the octopolar constant, $q_{3}$. Note that the 
solution $\psi^{I}$ is highly asymmetric due to the weight constant $\alpha$. For particles orbiting a central body deformed by this highly asymmetric multipolar field we might have instabilities, for simplicity we shall take the symmetric case, $\alpha=1 / 2$, in this case,

$$
\begin{aligned}
\delta \psi^{I} & =\frac{9}{4} a^{2} m q_{2} u^{5}+\frac{45}{4} 3 a^{3} m^{3} q_{2} u^{6}, \\
\delta \gamma^{I} & =\frac{15}{4} a^{2} m^{3} q_{2} u^{6} .
\end{aligned}
$$

In summary, we have that the discrepancies in the solutions appear in the fifth order of the inverse radial variable $u$ for the potential $\psi$ and the sixth order for the potential $\gamma$,

$$
\begin{aligned}
& \hat{\psi}=-\frac{q_{2}}{2} u^{3}-\frac{3}{2} m q_{2} u^{4}+\left(-\beta m^{2} q_{2}+\frac{3}{8} q_{4}\right) u^{5}+\mathcal{O}\left(u^{6}\right), \\
& \hat{\gamma}=-\frac{3}{4} m q_{2} u^{4}-3 m^{2} q_{2} u^{5}+\mathcal{O}\left(u^{6}\right) .
\end{aligned}
$$

with

$$
\beta^{N}=\frac{15}{4}, \beta^{E R Q}=\frac{24}{7}, \beta^{I}=\frac{3}{4}\left(5-3 a^{2}\right), \beta^{I I}=\frac{3}{56}\left(70-6 a^{2}\right) .
$$

For the differences up to the fifth order in $u$ we have, $\delta \gamma=\delta \hat{\gamma}=0$ and

$$
\delta \psi=\delta \hat{\psi}=-\delta \beta m^{2} q_{2} u^{5}
$$

with

$$
\delta \beta^{E R Q}=-\frac{9}{28}, \quad \delta \beta^{I}=-\frac{9}{4} a^{2}, \quad \delta \beta^{I I}=-\frac{9}{28} a^{2} .
$$

For general linear perturbations (not necessarily static axially symmetric) of the Schwarschild black hole we have the Regge-Wheeler [11] formalism. One can show [12] that the simple case of linear static axially symmetric perturbations in Weyl coordinates can also be put in the Regge-Wheeler form, 
as long as, the solutions do not have conic singularities. In the framework of above mentioned formalism one can also show that the dipolar perturbations can be eliminated by a transformation of coordinates as in the usual Newtonian case. This is not a trivial fact since relativistic dipolar moments are invariant tensorial quantities and as such cannot be made zero by a coordinate transformation. In the linear approximation the dipolar perturbations turn to be gauge dependent.

\section{An evolution equation for differences.}

A first and simple approach to the problem of looking for the discrepancies in the orbits of test particles moving around a deformed attraction center modeled with different relativistic gravitational fields (metrics) with the same Newtonian limit, is to compare circular orbits by means of an equation for the differences of the radial coordinate. The geodesic equation for the Weyl metric has three constants of motion, that in the plane $y=0$ determine completely the orbit of test particles:

$$
\begin{aligned}
& e^{2 \psi} \dot{t}^{2}-m^{2} e^{2 \gamma-2 \psi}\left(x^{2}-y^{2}\right)\left(\frac{\dot{x}^{2}}{x^{2}-1}+\frac{\dot{y}^{2}}{1-y^{2}}\right) \\
& \quad-m^{2} e^{-2 \psi}\left(x^{2}-1\right)\left(1-y^{2}\right) \dot{\varphi}^{2}=1, \\
& e^{2 \psi} \dot{t}=l, \\
& e^{-2 \psi}\left(x^{2}-1\right)\left(1-y^{2}\right) \dot{\varphi}=\frac{h}{m^{2}},
\end{aligned}
$$

where, as usual, $\dot{t}=d t / d s$, etc., $l$ and $h$ are constants related to the test particle energy and angular momentum, respectively.

From (66)-(68) we find the equation of the orbits in the equatorial 
plane, $y=0$,

$$
l^{2} e^{-2 \psi}-e^{2 \gamma+2 \psi} \frac{h^{2}}{m^{2}} \frac{x^{2}}{\left(x^{2}-1\right)^{3}} x^{\prime 2}-\frac{h^{2}}{m^{2}} \frac{e^{2 \psi}}{x^{2}-1}=1,
$$

with $x^{\prime}=\frac{d x}{d \varphi}$. It is more convenient to write this equation in terms of the potentials, $\hat{\psi}$ and $\hat{\gamma}$, and the variable $u=R_{S}^{-1}=[m(1+x)]^{-1}$. Hence,

$$
u^{\prime 2}+u^{2} e^{-2 \hat{\gamma}}-\frac{l^{2}}{h^{2}} e^{-2(\hat{\gamma}+2 \hat{\psi})}+\frac{1}{h^{2}} e^{-2(\hat{\gamma}+\hat{\psi})}-\frac{2 m u}{h^{2}} e^{-2(\hat{\gamma}+\hat{\psi})}-2 m u^{3} e^{-2 \hat{\gamma}}=0,
$$

that has the general form,

$$
u^{\prime 2}=F(u, \hat{\gamma}(u), \hat{\psi}(u))
$$

where

$$
F=e^{-2 \hat{\gamma}}\left(-u^{2}+2 m u^{3}+\frac{l^{2}}{h^{2}} e^{-4 \hat{\psi}}-\frac{1}{h^{2}} e^{-2 \hat{\psi}}+\frac{2 m}{h^{2}} u e^{-2 \hat{\psi}}\right) .
$$

By differentiation of (71) we obtain the general equation for the difference of the inverse radial coordinate of equatorial orbits,

$$
(\delta u)^{\prime}-\frac{1}{2 u^{\prime}} \frac{\partial F}{\partial u} \delta u=\frac{1}{2 u^{\prime}}\left(\frac{\partial F}{\partial \hat{\gamma}} \delta \hat{\gamma}+\frac{\partial F}{\partial \hat{\psi}} \delta \hat{\psi}\right)
$$

where $\delta \hat{\gamma}$ and $\delta \hat{\psi}$ represent the differences defined in (52).

Note that

$$
\frac{\partial F}{\partial \hat{\gamma}}=-2 F
$$

For circular orbits, $u=u_{0},\left(R_{S}=1 / u_{0}\right.$, the constant radius $)$, we have $F=0$. From (74) and (73) we get

$$
\delta u=-\left(\frac{\partial F}{\partial \hat{\psi}} / \frac{\partial F}{\partial u}\right)_{u_{0}} \delta \hat{\psi} .
$$


First, we shall compute the leading term in $u$ of this last equation, from (64) we find,

$$
\delta u=\frac{1}{3}\left(1-2 l^{2}\right) q_{2} u_{0}^{4} \delta \beta .
$$

This last equation in terms of the Schwarzschild like radial coordinate $R_{S}$ reads,

$$
-\delta R_{S}=\frac{1}{3}\left(1-2 l^{2}\right) q_{2} \frac{\delta \beta}{R_{S}^{2}}
$$

Then the difference decays with the square of the radius. It is also instructive to express equation $(76)$ in terms of the dimensionless parameter,

$$
\epsilon=\frac{\text { half Schwarzschild radius }}{\text { orbit radius }} \text {. }
$$

We get,

$$
\delta u=\delta \beta\left(1-2 l^{2}\right) \frac{q_{2}}{3 m^{3}} u_{0} \epsilon^{3} .
$$

For planets like Earth, Mercury, and Mars $\epsilon$ is a little parameter $\epsilon \sim 10^{-8}$. But, for a small test body orbiting around a neutron star we can have $\epsilon \sim$ $10^{-1}$. In this last case the multipolar fields can be originated, for instance, by polar jets of matter ejected by the neutron star. Finally, we want to comment that Eq. (75) gives a simple expression involving the exact metric.

\section{The perihelion shift.}

To compare the different multipole expansions we shall study the perihelion shift of a test particle orbiting in the equatorial plane of the deformed "black hole". In the order of approximation used in the present work we can put $e^{\hat{\psi}}=1+\hat{\psi}$ and $e^{\hat{\gamma}}=1+\hat{\gamma}$ in $(70)$ with no error,

$$
\begin{aligned}
& u^{\prime 2}+u^{2}-\frac{l^{2}-1}{h^{2}}-\frac{2 m u}{h^{2}}-2 m u^{3}= \\
& 2 \hat{\gamma}\left(u^{2}-\frac{l^{2}-1}{h^{2}}-\frac{2 m u}{h^{2}}-2 m u^{3}\right)+2 \hat{\psi}\left(-\frac{2 l^{2}}{h^{2}}+\frac{1}{h^{2}}-\frac{2 m u}{h^{2}}\right) .
\end{aligned}
$$


Doing $\hat{\psi}=\hat{\gamma}=0$ in the previous equation we obtain the well known equation for the motion of a test particle in the Schwarzschild spacetime.

Since the first term in the series expansion of the functions $\hat{\gamma}$ and $\hat{\psi}$ are proportional to $u^{4}$ and $u^{3}$, respectively [cf. Eqs. (44)-(51)], to use the orbital equation (80) without error we must limit the expansion of $\hat{\gamma}$ and $\hat{\psi}$ to the order seven and five in the variable $u$, respectively. Therefore we shall consider all the series up to the fifth order in $u$, that is the order wherein the first discrepancies between the different multipole expansions appear. From Eq. (80) we obtain,

$$
u^{\prime 2}+u^{2}=\left[l^{2}-1+2 m u+2 m h^{2} u^{3}+\frac{2}{3} q m^{3} u^{3}+\frac{1}{2} r m^{4} u^{4}+\frac{2}{5} s m^{5} u^{5}\right] / h^{2},
$$

where,

$$
\begin{aligned}
q & =\frac{3}{2}\left(2\left(l^{2}-1\right)+1\right) \frac{q_{2}}{m^{3}}, \\
r & =\left(15\left(l^{2}-1\right)+10\right) \frac{q_{2}}{m^{3}}, \\
s & =\frac{5}{2}\left[\left((4 \beta+6)\left(l^{2}-1\right)+2 \beta+9\right) \frac{q_{2}}{m^{3}}-\frac{3}{4}\left(2 l^{2}-1\right) \frac{q_{4}}{m^{5}}\right] .
\end{aligned}
$$

Note that $q, r$, and $s$ are dimensionless quantities. The semilatus rectum for the usual Keplerian orbit of a particle attracted by a central body with no multipolar deformations is, $p=h^{2} / m$. We shall use a dimensionless parameter similar to the homonymous one of the preceding section,

$$
\epsilon=\frac{m^{2}}{h^{2}}=\frac{m}{p}=\frac{\text { half Schwarzschild radius }}{\text { semilatus rectum }} .
$$

Note that for a circular orbit with $u=u_{0}$ we have $u_{0}=1 / p$. It is enlightening to write the equation (81) in terms of the new dimensionless variable $w=p u$,

$$
w^{\prime 2}+w^{2}=p^{2} \frac{l^{2}-1}{h^{2}}+2 w+2 \epsilon w^{3}+\frac{2}{3} q \epsilon^{2} w^{3}+\frac{1}{2} r \epsilon^{3} w^{4}+\frac{2}{5} s \epsilon^{4} w^{5} .
$$


By derivation we obtain,

$$
w^{\prime \prime}+w=1+3 \epsilon w^{2}+q \epsilon^{2} w^{2}+r \epsilon^{3} w^{3}+s \epsilon^{4} w^{4} .
$$

We remind that the discrepancies between the different multipole expansions are controlled by the parameter $\beta$ that it only appears in $s$ (last term). We shall search a solution of equation (87) in the form of a series expansion up to the fourth order in $\epsilon$,

$$
w=w_{0}+w_{1} \epsilon+w_{2} \epsilon^{2}+w_{3} \epsilon^{3}+w_{4} \epsilon^{4}
$$

From (87) we find the system of linear equations,

$$
\begin{aligned}
& w_{0}^{\prime \prime}+w_{0}=1, \\
& w_{1}^{\prime \prime}+w_{1}=3 w_{0}^{2}, \\
& w_{2}^{\prime \prime}+w_{2}=6 w_{0} w_{1}+q w_{0}^{2}, \\
& w_{3}^{\prime \prime}+w_{3}=3\left(2 w_{0} w_{2}+w_{1}^{2}\right)+2 q w_{0} w_{1}+r w_{0}^{3}, \\
& w_{4}^{\prime \prime}+w_{4}=6\left(w_{0} w_{3}+w_{1} w_{2}\right)+q\left(2 w_{0} w_{2}+w_{1}^{2}\right)+3 r w_{0}^{2} w_{1}+s w_{0}^{4} .
\end{aligned}
$$

The solution of this system can be expressed in terms of elementary functions, we find,

$$
\begin{aligned}
w_{0}= & 1+\mathrm{e} \cos \varphi \\
w_{1}= & 3\left(1+\mathrm{e}^{2} / 2\right)+3 \mathrm{e} \varphi \sin \varphi-\left(\mathrm{e}^{2} / 2\right) \cos 2 \varphi, \\
w_{2}= & 9\left(2+\mathrm{e}^{2}\right)+q\left(1+\mathrm{e}^{2} / 2\right)-(9 / 2) \mathrm{e}^{2} \cos \varphi-\left(3 \mathrm{e}^{2}+q \mathrm{e}^{2} / 6\right) \cos 2 \varphi \\
& +\left(3 \mathrm{e}^{3} / 16\right) \cos 3 \varphi+\left(27 \mathrm{e} / 2+15 \mathrm{e}^{3} / 4+q \mathrm{e}\right) \varphi \sin \varphi-3 \mathrm{e}^{2} \varphi \sin 2 \varphi, \\
w_{3}= & 135+81 \mathrm{e}^{2}+57 \mathrm{e}^{4} / 8+12 q+6 \mathrm{e}^{2} q+r+3 \mathrm{e}^{2} r / 2-\left(81 \mathrm{e} \varphi^{2} / 2\right. \\
& \left.+45 \mathrm{e}^{3} \varphi^{2} / 4+3 \mathrm{e} q \varphi^{2}\right) \cos \varphi-\left(27 \mathrm{e}^{2}+59 \mathrm{e}^{4} / 16+2 \mathrm{e}^{2} q+\mathrm{e}^{2} r / 2\right. \\
& \left.-9 \mathrm{e}^{2} \varphi^{2}\right) \cos 2 \varphi+\left(9 \mathrm{e}^{3} / 4+\mathrm{e}^{3} q / 8-\mathrm{e}^{3} r / 32\right) \cos 3 \varphi-\frac{1}{16} \mathrm{e}^{4} \cos 4 \varphi+
\end{aligned}
$$




$$
\begin{aligned}
& \left(189 \mathrm{e} \varphi / 2+135 \mathrm{e}^{3} \varphi / 4+9 \mathrm{e} q \varphi+5 \mathrm{e}^{3} q \varphi / 2+3 \mathrm{e} r \varphi / 2+3 \mathrm{e}^{3} r \varphi / 8\right. \\
& \left.-9 \varphi^{3} / 2\right) \sin \varphi-\left(63 \mathrm{e}^{2} \varphi / 2+15 \mathrm{e}^{4} \varphi / 4+2 \mathrm{e}^{2} q \varphi\right) \sin 2 \varphi \\
& +\frac{27}{16} \mathrm{e}^{3} \varphi \sin 3 \varphi \\
w_{4}= & 1134+810 \mathrm{e}^{2}+513 \mathrm{e}^{4} / 4+135 q+81 \mathrm{e}^{2} q+57 \mathrm{e}^{4} q / 8+2 q^{2}+\mathrm{e}^{2} q^{2} \\
& +15 r+18 \mathrm{e}^{2} r+15 \mathrm{e}^{4} r / 8+s+3 \mathrm{e}^{2} s+3 \mathrm{e}^{4} s / 8-\left(2997 \mathrm{e}^{2} / 8\right. \\
& +1215 \mathrm{e}^{3} \varphi^{2} / 8+225 \mathrm{e}^{5} \varphi^{2} / 32+81 \mathrm{e} q \varphi^{2} / 2+45 \mathrm{e}^{3} q \varphi^{2} / 4+\mathrm{e}^{2} \varphi^{2} / 2 \\
& \left.+9 \mathrm{e} \varphi^{2} / 2+9 \mathrm{e}^{3} r \varphi^{2} / 8-27 \mathrm{e} \varphi^{4} / 8\right) \cos \varphi+\left(-270 \mathrm{e}^{2}-531 \mathrm{e}^{4} / 8\right. \\
& -27 \mathrm{e}^{2} q-59 \mathrm{e}^{4} q / 16-\mathrm{e}^{2} q^{2} / 3-6 \mathrm{e}^{2} r-31 \mathrm{e}^{4} r / 32-\mathrm{e}^{2} s-\mathrm{e}^{4} s / 6+ \\
& \left.135 \mathrm{e}^{2} \varphi^{2}+45 \mathrm{e}^{4} \varphi^{2} / 2+9 \mathrm{e}^{2} q \varphi^{2}\right) \cos 2 \varphi+\left(27 \mathrm{e}^{3}+711 \mathrm{e}^{5} / 256+9 \mathrm{e}^{3} q / 4+\right. \\
& \left.\mathrm{e}^{3} q^{2} / 48+3 \mathrm{e}^{3} r / 16-\mathrm{e}^{3} s / 8-243 \mathrm{e}^{3} \varphi^{2} / 32\right) \cos 3 \varphi-\left(9 \mathrm{e}^{4} / 8+\mathrm{e}^{4} q / 16\right. \\
& \left.-\mathrm{e}^{4} r / 32+\mathrm{e}^{4} s / 120\right) \cos 4 \varphi+\frac{5}{256} \mathrm{e}^{5} \cos 5 \varphi+\left(6237 \mathrm{e} \varphi / 8+5 \mathrm{e}^{3} q^{2} \varphi / 12\right. \\
+ & 2835 \mathrm{e}^{3} \varphi / 8+1455 \mathrm{e}^{5} \varphi / 64+189 \mathrm{e} q \varphi / 2+135 \mathrm{e}^{3} q \varphi / 4+3 \mathrm{e}^{2} \varphi / 2 \\
+ & 33 \mathrm{e} r \varphi / 2+69 \mathrm{e}^{3} r \varphi / 8+2 \mathrm{e} s \varphi+3 \mathrm{e}^{3} s \varphi / 2-243 \mathrm{e} \varphi^{3} / 4-135 \mathrm{e}^{3} \varphi^{3} / 8 \\
& \left.-9 \mathrm{e} q \varphi^{3} / 2\right) \sin \varphi-\left(675 \mathrm{e}^{2} \varphi / 2+627 \mathrm{e}^{4} \varphi / 8+63 \mathrm{e}^{2} q \varphi / 2+15 \mathrm{e}^{4} q \varphi / 4\right. \\
+ & \left.\mathrm{e}^{2} q^{2} \varphi / 3+9 \mathrm{e}^{2} r \varphi / 2+3 \mathrm{e}^{4} r \varphi / 8-18 \mathrm{e}^{2} \varphi^{3}\right) \sin 2 \varphi+\left(891 \mathrm{e}^{3} \varphi / 32\right. \\
+ & \left.135 \mathrm{e}^{5} \varphi / 64+27 \mathrm{e}^{3} q \varphi / 16-9 \mathrm{e}^{3} r \varphi / 32\right) \sin 3 \varphi-\frac{3}{4} \mathrm{e}^{4} \varphi \sin 4 \varphi
\end{aligned}
$$

Only the function $w_{4}$ contains the parameter $s$.

The perihelion, as well as, the aphelion of the test particle orbit are given by extremals of the function $u$ or $w$. In (90) we chose the constants of integration to have $\varphi=0$ in the position of the perihelion at any order in $\epsilon$. The aphelion is nearby $\varphi=\pi$ and the next perihelion closed to $\varphi=2 \pi$. The integration constant e is the orbit eccentricity.

We shall begin the computation of the perihelion shift in the lower 
order in $\epsilon$. We have

$$
w=w_{0}+\epsilon w_{1},
$$

where $w_{0}$ and $w_{1}$ are given in (90). We verify that, $w^{\prime}(0)=0$. Therefore, the perihelion is located the in the angular position $\varphi=0$; the next perihelion is in $\varphi=2 \pi+L$, where $L$ is a small unknown quantity. We have by definition, $w^{\prime}(2 \pi+L)=0$. Expanding the function $w^{\prime}(\varphi)$ up to the first order in $L$ in the neighborhood of $2 \pi$, we find

$$
w^{\prime}(2 \pi+L)-w^{\prime}(2 \pi)=L w^{\prime \prime}(2 \pi)+\mathcal{O}\left(L^{2}\right) .
$$

We obtain the advance of the perihelion up to the first order in $\epsilon, L=6 \pi \epsilon$, i.e., the usual result for a particle orbiting in the Schwarzschild metric. Now, $w$ up to the four order in $\epsilon$ is given by the series ( 88$)$. From the previous result we know that the series expansion of $L$ begins with the order $\epsilon$. Thus, we will look for a constant $L$ of the form,

$$
L=L_{1} \epsilon+L_{2} \epsilon^{2}+L_{3} \epsilon^{3}+L_{4} \epsilon^{4},
$$

solution of the equation

$$
w^{\prime}(2 \pi+L)-w^{\prime}(2 \pi)=L w^{\prime \prime}(2 \pi)+\frac{L^{2}}{2} w^{\prime \prime \prime}(2 \pi)+\frac{L^{3}}{6} w^{\prime \prime \prime \prime}(2 \pi)+\mathcal{O}\left(L^{4}\right) .
$$

Since the fifth derivative is of order $\epsilon$ we cut the expansion in the fourth derivative. Also, we note that $w^{\prime}(0)=w^{\prime}(2 \pi+L)=0$. By identification of the coefficients of the different powers of $\epsilon$ in the series expansion of the two member of Eq. (94) we obtain,

$$
\begin{aligned}
& L_{1}=6 \pi, \\
& L_{2}=\frac{1}{2} \pi\left(90+15 \mathrm{e}^{2}+4 q\right),
\end{aligned}
$$




$$
\begin{gathered}
L_{3}=\pi\left(1620+450 \mathrm{e}^{2}+120 q+20 \mathrm{e}^{2} q+12 r+3 \mathrm{e}^{2} r\right) / 4, \\
L_{4}=\pi\left(379080+145800 \mathrm{e}^{2}+7065 \mathrm{e}^{4}+38880 q+10800 \mathrm{e}^{2} q+480 q^{2}\right. \\
\left.+80 \mathrm{e}^{2} q^{2}+4896 r+2088 \mathrm{e}^{2} r+384 s+288 \mathrm{e}^{2} s\right) / 96 .
\end{gathered}
$$

We see that the discrepancies between the different multipole expansions are reflected in $L_{4}$ via $s$. So until the order $\epsilon^{3}$ we have the same perihelion shift for the multipole expansions considered in the present paper. Moreover, Eqs. (93) and (96) with $q=r=s=0$ gives us the perihelion shift of an orbit in Schwarzschild geometry up to the fourth order in $\epsilon$. We have that the contribution to the perihelion due to deformation of the spherically symmetric attraction center appears in the second order in $\epsilon$; a fact that is well known in Newtonian theory.

Now, let us return to the difference equation (73). For $\hat{\psi}$ and $\hat{\gamma}$ we use the solutions up to the five order in $u$. Therefore we have, $\delta \hat{\gamma}=0$ and $\delta \psi$ given by (64). The equation (73) reduces to,

$$
u^{\prime}(\delta u)^{\prime}-\frac{1}{2}\left(\frac{2}{p}-2 u+6 m u^{2}+\mathcal{O}(\epsilon)\right) \delta u=\delta \beta\left(2 l^{2}-1\right) \frac{q_{2}}{m^{3}} p^{3} \epsilon^{4} u^{5}
$$

In this equation we can replace the solution $u$, or equivalently $w$, by his first order approximation $w_{0}=1+\operatorname{e} \cos \varphi$. We find for the lower order in $\epsilon$,

$\mathrm{e} \sin \varphi(\delta u)^{\prime}-\left[\mathrm{e} \cos \varphi-\frac{3 m}{p}(1+\mathrm{e} \cos \varphi)^{2}\right] \delta u=-\delta \beta\left(2 l^{2}-1\right) \frac{q_{2}}{m^{3}} \frac{1}{p} \epsilon^{4}(1+\mathrm{e} \cos \varphi)^{5}$

By letting $\mathrm{e} \rightarrow 0$ in the previous equation we recover the equation for the differences of circular orbits, Eq. (79). Since Eq. (98) is a linear first order differential equation, its solution has a simple integral representation that in this case is rather useless due to the fact that the integrals are not elementary. The nonhomogeneous part of the solution is proportional to $\epsilon^{4}$. Fact that 
confirms that the first discrepancies between the different expansions are proportional to $\epsilon^{4}$.

\section{Discussion}

From the study of particular cases, we believe, that are representative of different possible series expansions of the metric functions for Weyl solutions that have the same Newtonian limit. We found a strong indication that the use of the right relativistic multipolar expansion to describe a deformed body need to be considered only in the case of a strong gravitational regime like bodies orbiting close (a few Schwarzschild radius) around a very compact object like a neutron star. For usual planetary motion, in particular for the perihelion shift, the effect of having different multipolar expansions with the same Newtonian limit can be completely ignored. Also when studying the stability of orbits (chaos) of particles moving around deformed bodies, one of us [7] found no difference in the trajectories for Newtonian and ERQ deformations. Recently, the same effect was study on the motion of a gyroscope [18].

\section{Acknowledgments}

P.S.L. thanks CNPq and FAPESP for financial support and S.R. Oliveira for discussions, also acknowledges the warm hospitality of the "Laboratoire". 


\section{References}

[1] D. Merrit, Science 271, 337 (1996)

[2] See for instance, H. Robertson and T. Noonan, "Relativity and Cosmology" (Saunders, London 1968) pp 272-278.

[3] K. Thorne, Rev. Mod. Phys. 52, 299 (1980).

[4] R. Geroch, J. Math. Phys. 11, 2580 (1970).

[5] R.O. Hansen, J. Math. Phys. 15, 46 (1974).

[6] Y. Gürsel, Gen. Rel. Grav. 12, 1003 (1983).

[7] E. Guéron and P.S. Letelier, Phys. Rev. E 63, 035201 (2001).

[8] See for instance, Ya. B. Zeldovich and I.D. Novikov, "Relativistic Astrophysics" (University of Chicago Press, Chicago 1971) pp 130-134; M. Carmeli, "Classical Fields: General Relativity and Gauge Theory" (John Wiley, New York 1982) pp 177-182.

[9] F. Bertola et al. Ap. J. 458, L67 (1996).

[10] H. Quevedo, Phys. Rev. D 392904 (1989).

[11] T. Regge and J.A. Wheeler, Phys. Rev. 108, 1063 (1957).

[12] W.M. Vieira and P.S. Letelier, Ap. J. 513, 383 (1999).

[13] C. Hoenselaers, in "Gravitation Collapse and Relativity, H. Sato and T. Nakamura Eds. (World Scientific, Singapore, 1986) pp 176-184. 
[14] J. Ehlers, in "Grundlagenprobleme der Modernen Physik", A. Erdélyi, J.Pfarr, and E.-W. Stachov, Eds. (BI-Verlag, Mannheim, 1981) pp 6584.

[15] G. Erez and N. Rosen, Bull. Res. Counc. Isr. 8F, 47 (1959).

[16] P.S. Letelier, Class. Q. Grav., 16, 1207 (1999).

[17] V.S. Manko, Class. Q. Grav., 7, L209 (1990)

[18] L. Herrera and J.L. Hernández Pastora, J. Math. Phys. 41, 7544 (2000). 


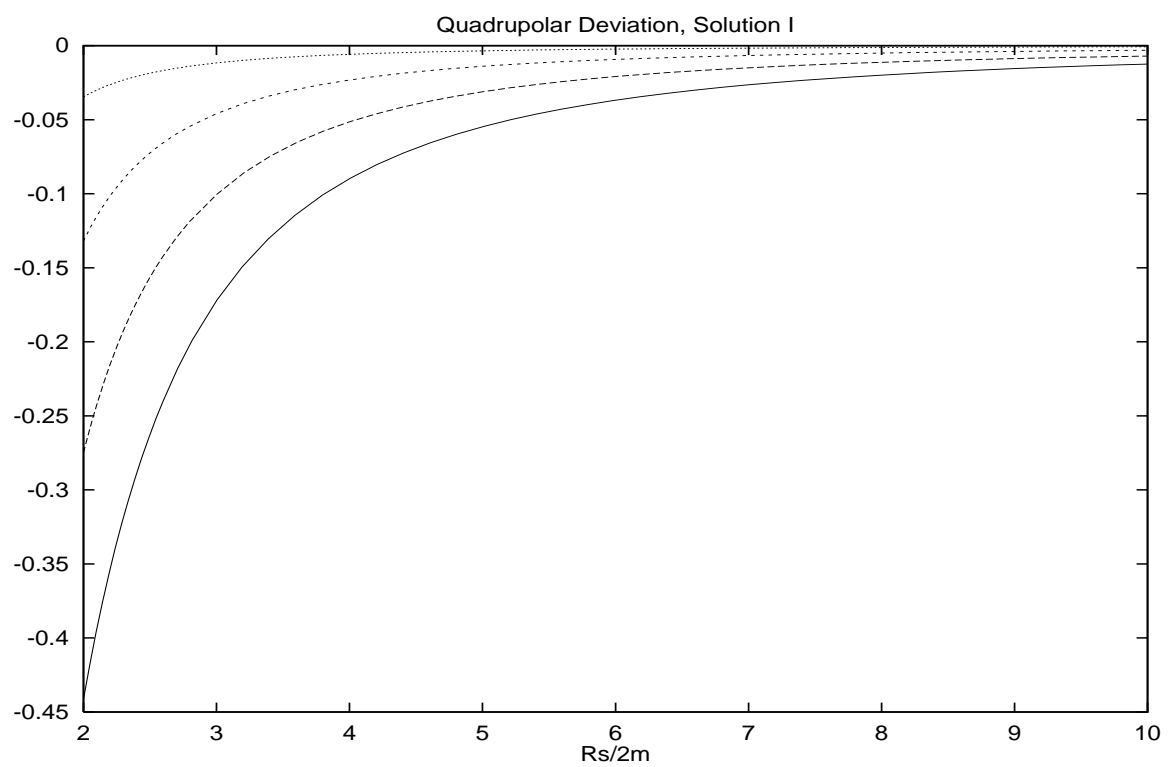

Figure 1: We present the deviation for $\psi_{2}=\psi_{2}^{I}$ on the plane $\vartheta=\pi / 2$ for $a=1$, and different values of the constant $\alpha: 0.25$ (top curve), $0.5,0.75,1.0$ (bottom). 


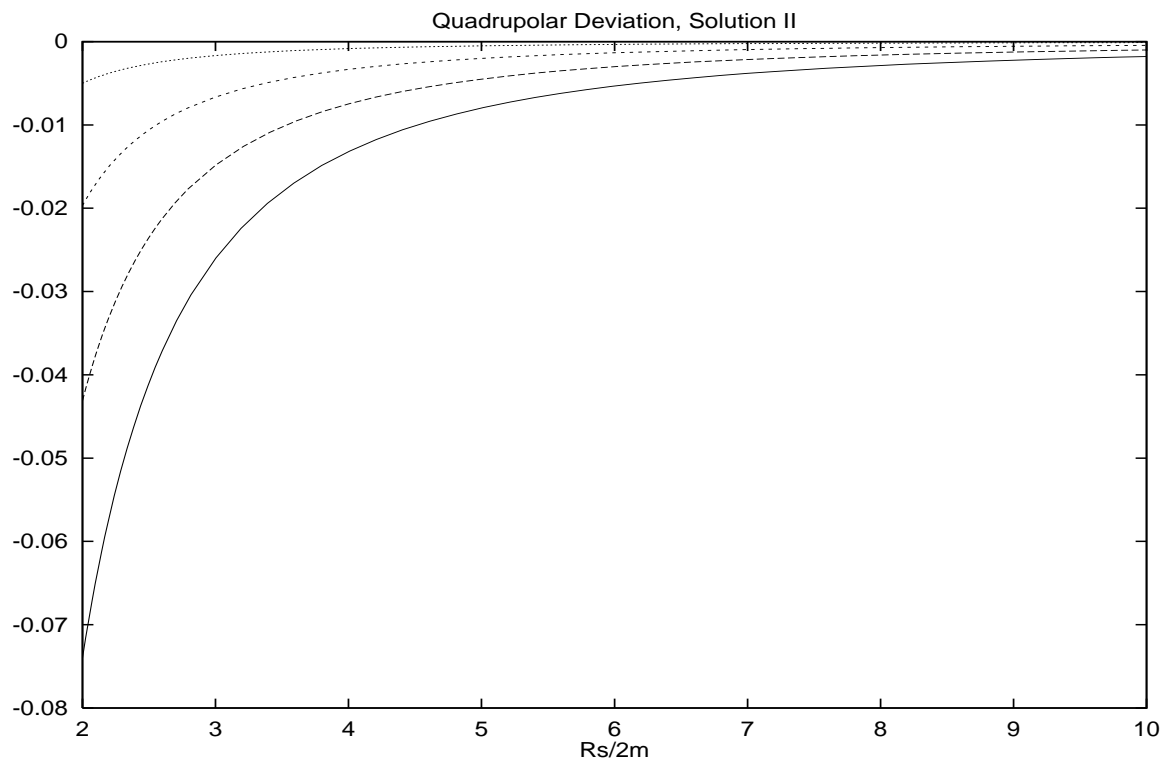

Figure 2: We show the deviation of $\psi_{2}=\psi_{2}^{I I}$ on the plane $\vartheta=\pi / 2$ for different values of the parameter $a$ : 0.25 (top curve), 0.5, 0.75, 1.0 (bottom). The case $a=1$ corresponds to the ERQ solution. 